\title{
Feasibility of Patient Specific Aortic Blood Flow CFD Simulation
}

\author{
Johan Svensson $^{1,5}$, Roland Gårdhagen ${ }^{1,5}$, Einar Heiberg ${ }^{2}$, Tino Ebbers ${ }^{3,5}$, \\ Dan Loyd ${ }^{1}$, Toste Länne ${ }^{3,5}$, and Matts Karlsson ${ }^{4,5}$ \\ ${ }^{1}$ Department of Mechanical Engineering, Linköping University, Sweden, \\ johsv@ikp.liu.se \\ ${ }^{2}$ Department of Clinical Physiology, Lund University, Sweden \\ ${ }^{3}$ Department of Medicine and Care, Linköping University, Sweden \\ ${ }^{4}$ Department of Biomedical Engineering, Linköping University, Sweden \\ ${ }^{5}$ Center for Medical Image Science and Visualization (CMIV), \\ Linköping University, Sweden
}

\begin{abstract}
Patient specific modelling of the blood flow through the human aorta is performed using computational fluid dynamics (CFD) and magnetic resonance imaging (MRI). Velocity patterns are compared between computer simulations and measurements. The workflow includes several steps: MRI measurement to obtain both geometry and velocity, an automatic levelset segmentation followed by meshing of the geometrical model and CFD setup to perform the simulations follwed by the actual simulations. The computational results agree well with the measured data.
\end{abstract}

\section{Introduction}

The aorta is the primary blood vessel for transporting blood from the left heart to the systemic circulation. It has a very complex three-dimensional geometry including curving, branching and tapering. To be able to describe this very complex flow situation in detail in the normal human as well as for different pathological conditions in various diseases, a detailed description of the flow situation is crucial.

One common systemic disorder that can be studied with the use of detailed velocity information is atherosclerosis. Atherosclerosis is the main reason for many cardiovascular diseases [1]. However, atherosclerosis is not uniformly localized which indicates an influence from the blood velocity field on the genesis of atherosclerosis 2]. The near wall flow of the artery has thus been in focus, often in the form of wall shear stress (WSS) i.e. the frictional load on the artery wall. WSS have been intensively used to investigate the correlation with atherosclerosis all the way back to the first studies by 3] and [4. Although there have been attempts to estimate WSS from direct flow measurements [5] the process is still inherently hampered with the need to make critical assumptions on the flow profile and three dimensional phase contrast magnetic resonance imaging (PC-MRI) velocity measurements lack the necessary resolution in the near 


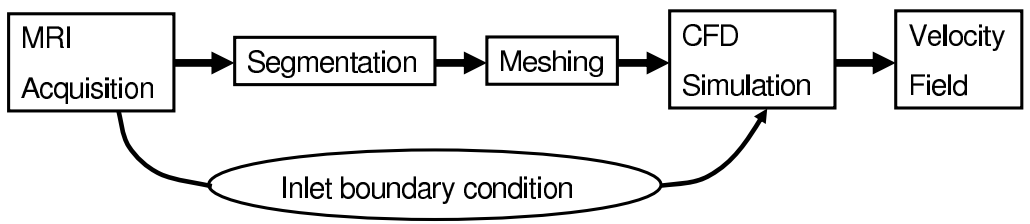

Fig. 1. Workflow overview

wall region. Thus, there are currently no non-invasive methods that can reliably be used to compute WSS directly to study or monitor the progress of the atherosclerosis.

To circumvent this CFD simulations are used to obtain a much more detailed view of the blood flow situation near the wall in the arteries. Using CFD techniques in the cardiovascular system have been made by numerous of research groups e.g. 6, 7, 8, 9, 10, and is constantly beeing refined and developed. However, creating a patient specific flow simulation model of a human aorta includes a wide range of engineering and medical disciplines. Some important parts are: state-of-the-art imaging techniques with sufficient resolution and quality, fast and accurate segmentation methods to gain a correct geometry and the very latest in CFD technology.

As a validation the flow simulation results are compared with 2D MRI velocity measurements. This kind of validation between CFD and measured MRI velocities have previously been performed by other groups before [11, 12 but studies conducted on patient specific models on large arteries in-vivo are rare [12,13.

To be able to use the proposed approach in a clinical situation all the steps need to be highly automated and fast. The aim of this work is to demonstrate the feasibility to produce accurate patient specific flow simulations in the human aorta that can be used in a clinical situation.

\section{Method}

The workflow is outlined in Figure 1. Starting from the left we need MRI measurement to obtain both geometry and velocity, an automatic levelset segmentation followed by meshing (the geometrical model) and finally the CFD setup to perform the simulations. The method is described in cronological order.

\subsection{MRI Acquisition}

Magnetic Resonance Imaging was used to collect data from 2 male volunteers (20 denoted (I) and 25 denoted (II) year) using a 1.5 T MRI scanner (Philips Achieva, Philips Medical Systems, Best, the Netherlands). Geometrical information of the complete aorta was obtained within a breath hold using a $3 \mathrm{D}$ gadoliniumenhanced gradient-echo sequence (TE $1.6 \mathrm{~ms}$, TR $5.3 \mathrm{~ms}$, and flip angle 40, field of view 400x360x80 mm, acquisition matrix 400x207x80, SENSE factor 1.5). The $30 \mathrm{ml}(0.5 \mathrm{mmol} / \mathrm{ml})$ contrast bolus (Omniscan, Amersham Health, Oslo, 
Norway) was injected at $2.0 \mathrm{ml} / \mathrm{s}$. Randomly segmented central k-space ordering (CENTRA) was used. The three-dimensional volume data was reconstructed to a resolution of $0.78 \times 0.78 \times 1.00 \mathrm{~mm}$.

Time-resolved information of the aortic flow velocities were obtained by performing a through-plane $2 \mathrm{D}$ velocity $\mathrm{MRI}$ acquisition (echo time $2.3 \mathrm{~ms}$, repetition time $3.9 \mathrm{~ms}$, flip angle 15 , velocity encoding range $2.00 \mathrm{~m} / \mathrm{s}$ ) placed supracoronary perpendicular to the flow direction. The acquisition was performed during a breath hold using SENSE factor 2.0 and retrospective cardiac gating to a vectorcardiogram (VCG). The $10 \mathrm{~mm}$ thick slice was acquired with a field of view of $350 \times 297 \mathrm{~mm}$, acquisition matrix 144x122, and a temporal resolution of $31.2 \mathrm{~ms}$ (I) or $39 \mathrm{~ms}$ (II). The acquired data were reconstructed to 40 timeframes per heart cycle with a spatial resolution of $1.37 \times 1.37 \mathrm{~mm}$. The velocity data were corrected for effects of concomitant gradient fields and eddy currents.

\subsection{Segmentation}

The purpose of the segmentation is to create a detailed three-dimensional geometric description of the aorta that can be used for meshing. The accuracy of the segmentation is crucial for the final CFD simulation result [14, 15, 16. We used a fast level set algorithm originally proposed by [17]. In a level set approach one starts with a small seed point and an implicit surface is allowed to expand outwards [18]. The expansion speed is determined by a speed image and the local curvature. The speed image was calculated as:

$$
\mathbf{I}_{\text {Speed }}(\boldsymbol{x})=\alpha_{0} \mathbf{I}(\boldsymbol{x})-\alpha_{1} \mathbf{I}_{\text {Edge }}(\boldsymbol{x})-\bar{I}_{\text {Seed }}+\alpha_{2}
$$

where $\mathbf{I}_{\text {Speed }}$ is speed image, $\boldsymbol{x}$ indicates spatial dependency, $\alpha_{n}$ are user adjusted coefficients, $\mathbf{I}$ is the MRI image, $\bar{I}_{\text {Seed }}$ is mean intensity of the seed points, and $\mathbf{I}_{E d g e}$ is an edge image created by sobel filtering. The value of the coefficients were not very critical. They were roughly set to $\alpha_{0}=7, \alpha_{1}=8$, and $\alpha_{2}=0$.

The expansion speed were calculated as:

$$
F(\boldsymbol{x})=\mathbf{I}_{\text {Speed }}(\boldsymbol{x})+\beta \boldsymbol{\kappa}(\boldsymbol{x})
$$

where $F$ is the local expansion speed, $\mathbf{I}_{\text {Speed }}$ is the speed image, $\beta$ is a fix coefficient set to 0.45 , and $\kappa$ finally is the local curvature of the level set surface.

By using a virtual 3D-pen with variable thickness the user could place seed points and at some places force the expansion velocity to zero. Manual intervention were typically needed to avoid the heart and pulmonary artery. The total time for the segmentation was around one hour per data set, but might be significantly shortened by improved user interface and practice. The used levelset algorithm is essentially a fast marching algorithm with an approximation to calculate the local curvature. This allows the algorithm to be comparable in speed with fast marching algorithms but still use curvature to produce a smoother result. The algorithm is binary so the final result is a binary mask of the aorta.

The algorithm was implemented into a cardiac image analysis software package (http://segment.heiberg.se/). 
Model I

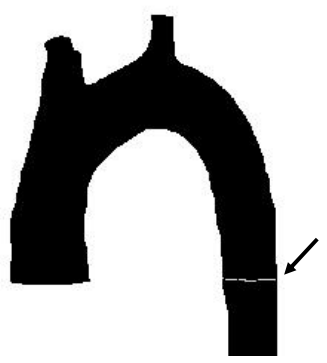

Model II

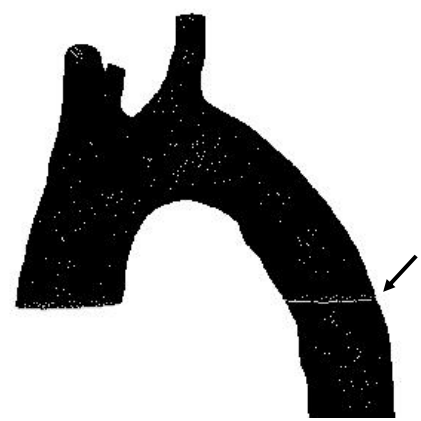

Fig. 2. Meshes of the aorta model I (left) and Model II (right). Location of the comparision cross-sections are marked by the arrows.

\subsection{Meshing}

The first step is to smooth the binary result from the level set algorithm. This was done by applying a 3D Gaussian smoothing filter. The radius of the filter was set to $2 \mathrm{~mm}$ where the distance denotes the point where the energy of the filter has dropped to $1 / e$. An isosurface was created using the marching cubes algorithm from the smoothed result of the binary mask with the iso level of 0.5. The isosurface was then converted to a sterolithography format (.STL). This file could the be imported by the mesh generator ICEM 10.0. Due to the smoothing procedure the geometry at the very proximal parts of inlets and outlets were changed so these parts were cropped of to ensure a correct geometry. An unstructured mesh was applied to the geometry, this choice was made because of the complexity in the geometry. A more quad element based mesh can be used but requiring more manual intervention. The meshes created are outlined in Figure 2,

Because that interesting areas for atherosclerosis are near the artery wall the mesh near the wall was refined by inserting four prism layers, see Figure 3 .

\subsection{CFD Simulation}

The CFD simulations are performed with the commercial software Fluent version 6.1.18, which uses a finite volume method. The governing equations are the Navier-Stokes equations consisting of the continuity equation, Equation 3, and the momentum equations, Equation 4 .

$$
\begin{gathered}
\oint \rho \mathbf{V} \cdot d \mathbf{A}=0 \\
\oint \mathbf{V V} \cdot d \mathbf{A}=-\oint p \mathbf{I} \cdot d \mathbf{A}+\oint \tau \cdot d \mathbf{A}
\end{gathered}
$$




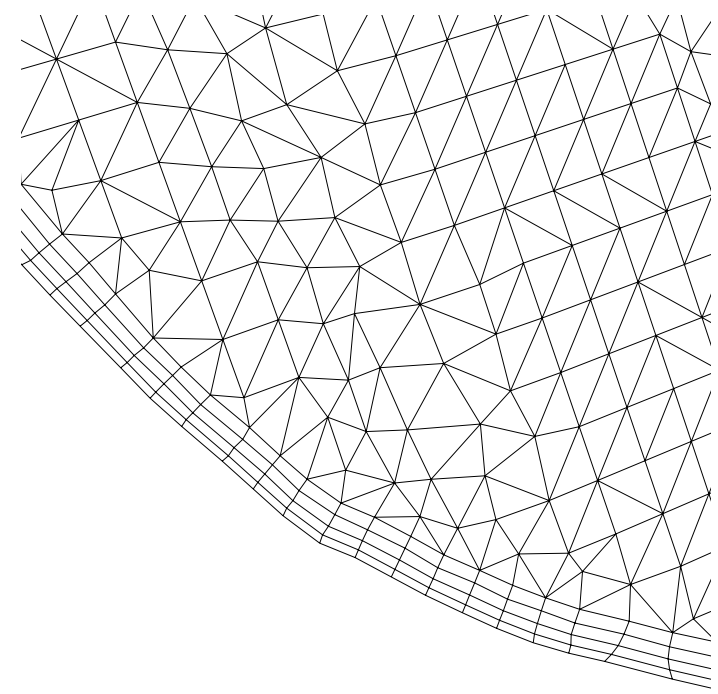

Fig. 3. Prism layers at the inlet in one of the models

where $\mathbf{V}$ is the velocity vector, $\rho$ is the density, $p$ is the pressure, $\mathbf{I}$ is the identity matrix, and $\tau$ is the stress tensor. The density of the fluid was set to $1060 \mathrm{~kg} / \mathrm{m}^{3}$ and the viscosity to $0.00345 \mathrm{Ns} / \mathrm{m}^{2}$.

The velocity profile is measured with $2 \mathrm{D}$ MRI in a cross section in the ascending aorta and used as the inflow boundary condition. The outflow boundary conditions ensure conservation of mass. $15 \%$ of the flow is assumed to leave the model through the first branch (brachiosephalic trunk), 5\% through the second branch (left common carotid), 10\% through the third branch (left subclavian artery), and $70 \%$ exits through the abdominal outflow. The arterial wall in the model is treated as rigid.

\section{Results and Discussion}

The results of the CFD simulation were evaluated in a cross-section in the decending aorta where we both had CFD velocity field and 2D MRI velocities. The results used are at three different times when the flow accellerates $\left(t_{1}\right)$ near the peak velocity $\left(t_{2}\right)$ and at the deccelaration $\left(t_{3}\right)$ phase. Both measured and CFD simulated velocities are seen in Figure 4.

From MRI images through segmentation and meshing procedure it takes less then two hours and the result is a suffiently accurate patient specific mesh ready for CFD simulations.

A good agreement is found between the stationary CFD simulation results to the PC-MRI measured velocities (Figure 41). Both flow profile and the velocity magnitude shows good agreement. There are difference as well that may depend on the chosen outflow in the aortic arch branches. 


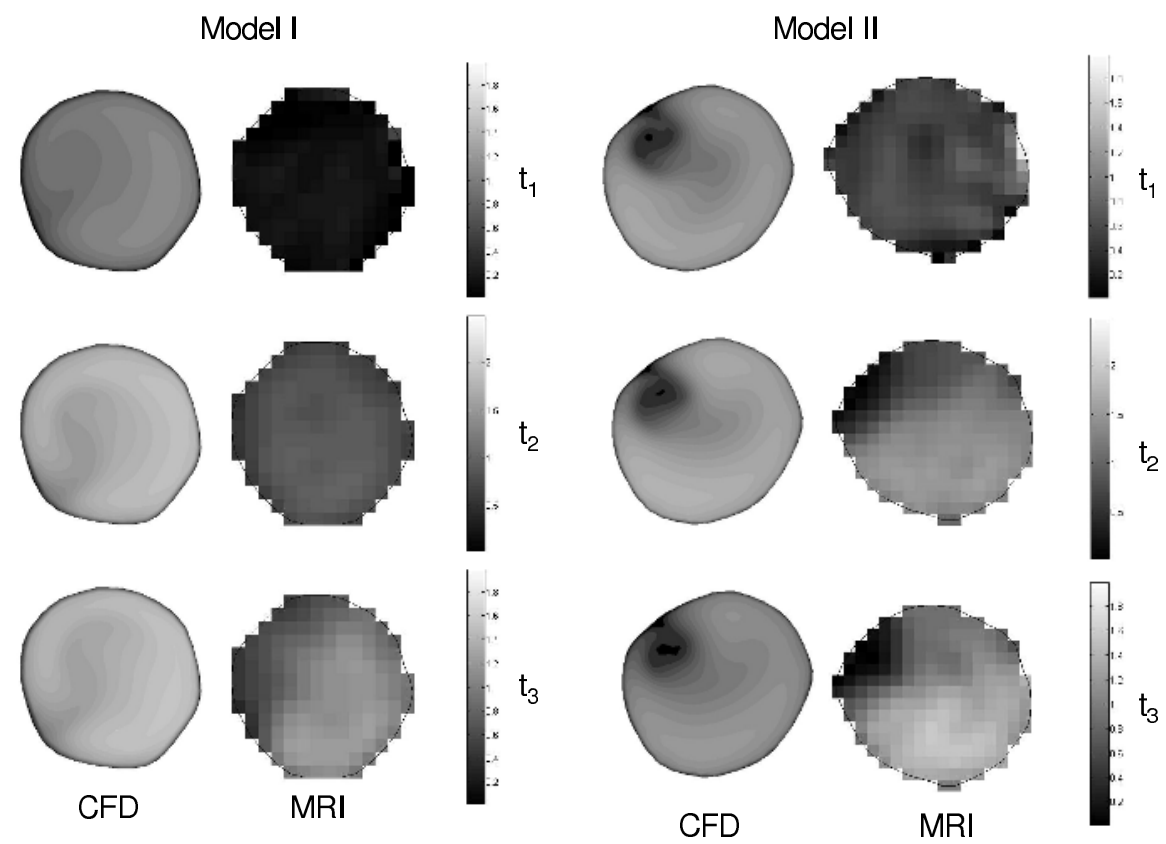

Fig. 4. Velocity magnitude in a decending aorta cross-section from both PC-MRI measurement and CFD simulations. Time position $t_{1}, t_{2}$ and $t_{3}$ correspond to accelerating flow, peak velocity and deaccelerating flow.

Using the described setup it is fully feasible with todays technology to scan a patient in the afternoon, and have an accurate flow simulation the following morning.

Future research will focus on further improvement to the segmentation process to further minimize the need for user interaction. One possible improvement would be to use template based method for segmentation. We also plan to perform time resolved CFD simulation, and to do larger studies involving larger number of subjects.

\section{References}

1. Berenson, G., Srinivas, S., Bao, W., Newman III, W., Tracy, R., Wattingney, W.: Association between multiple cardiovascular risk factors and atherosclerosis in childeren and young adults. The New England Journal of Medicine 338 (1998) $1650-1656$

2. Humprey, J.: Cardiovascular Solid Mechanics. Springer-Verlag (2002)

3. Caro, C., Fitz-Gerald, J., Schroter, R.: Arterial wall shear: Observation, correlation and proposal of a shear dependent mass transfer mechanism for atherogenisis. In: Procedings of Royal Society in London. Volume B:177. (1971) 109-159

4. Fry, D.: Acute vascular endothelial changes associated with increased blood velocity gradient. Circular Research 22 (1968) 165-197 
5. Wu, S., Ringgaard, S., Oyre, S., Hansen, M., Rasmus, S., Pedersen, E.: Wall shear rates differ between the normal carotid, femoral, and bracial arteries: An in vivo MRI study. Journal of Magnetic Resonance Imaging (2004) 188-193

6. Quarteroni, A., Tuveri, M., Veneziani, A.: Computational vascular fluid dynamics: problems, models and methods. Computing and Visualization in Science 2 (2000) 163-197

7. Cebral, J., Castro, M., Soto, O., Löhner, R., Alperin, N.: Blood-flow models of the circle of willis from magnetic resonance data. Journal of Engineering Mathematics 47 (2003) 369-386

8. Finol, E., Amon, C.: Flow-induced wall shear stress in abdominal aortic aneurysms: Part i - steady flow hemodynamics. Computer Methods in Biomechanics and Biomedical Engineering 5 (2002) 309-318

9. Finol, E., Amon, C.: Flow-induced wall shear stress in abdominal aortic aneurysms: Part ii - pulsatile flow hemodynamics. Computer Methods in Biomechanics and Biomedical Engineering 5 (2002) 319-328

10. Steinman, D., Thomas, J., Ladak, H., Milner, J., Rutt, B., Spence, J.: Reconstruction of carotid bifurcation hemodynamics and wall thickness using computational fluid dynamics and mri. Magnetic Resonance in Medicine 47 (2002) 149-159

11. Glor, F., Westenberg, J., Vierendeels, J., Danilouchkine, M., Verdonck, P.: Validation of the coupling of magnetic resonance imaging velocity measurement with computational fluid dynamics in an U bend. Artificial Organs 27 (2002) 622-635

12. Ku, J., Elkins, C., Taylor, C.: Comparison of CFD and MRI flow and velocities in an in vitro large artery bypass graft model. Annals of Biomedical engineering $\mathbf{3 3}$ (2005) 257-269

13. Leuprecht, A., Perktold, K., Kozerke, S., Boesiger, P.: Combined cfd and mri study of blood flow in a human ascending aorta model. Biorheology 39 (2002) 425-429

14. Friedman, M., Deters, O., Mark, F., Bargeron, C., Hutchins, G.: Arterial geometry affects hemodynamics. A potential risk factor for atherosclerosis. Atherosclerosis 46 (1983) 225-231

15. Svensson, J., Gårdhagen, R., Karlsson, M.: Geometrical considerations in patient specific models of a human aorta with stenosis and aneurysm. In: Computational Fluid Dynamics 2004 Proceedings of the Third International Conference on Computational Fluid Dynamics, ICCFD3, Toronto, 12-16 July 2004, ISBN: 3-54031800-3. (2004)

16. Svensson, J., Gårdhagen, R., Karlsson, M.: Assesment of geometrical influence on wss estimation in the human aorta. Accepted for publication in: WSEAS Transactions on Fluid Mechanics (2006)

17. Nilsson, B., Heyden, A.: A fast algorithm for level set-like active contours. Pattern Recognition Letters 24 (2003) 1331-1337

18. Sethian, J.A.: Level set methods and fast marching methods. Cambridge University Press, Cambridge, USA (1999) ISBN 0521645573. 